\title{
Accomplishments in Integrated Nonlinear Optics using Silicon Microring CROWs (Project Report 0642603-Y5)
}

\section{SHAYAN MOOKHERJEA}

Department of Electrical and Computer Engineering, University of California, San Diego, MC 0407 La Jolla CA 92093-0407 USA

Email:smookher@ucsd.edu

\section{Introduction and Goals}

Coupled resonator optical waveguides (CROWs), proposed as a type of integrated optics device in 1998-1999 by Yariv et al. [1] and Stefanou and Modinos [2], are linear sequences of micro-resonators fabricated on a chip that guide light from one end of the chain to the other by nearest-neighbor coupling. Figure 1 shows the operating principle of the CROW. Two identical microresonators when coupled show, under optical excitation, a split resonance consisting of two supermodes. The separation between the modes is proportional to the magnitude of the coupling coefficient $\kappa$ (which appears in the matrix formulation of the coupled-mode equations). A CROW consisting of a chain of $\mathrm{N}$ coupled resonators shows an $\mathrm{N}$-fold splitting of the transmission spectrum. Whether the individual peaks are resolvable depends on the strength of the inter-resonator coupling, the loss, and the exact distribution of coupling coefficients along the chain (i.e., apodization).

(a)
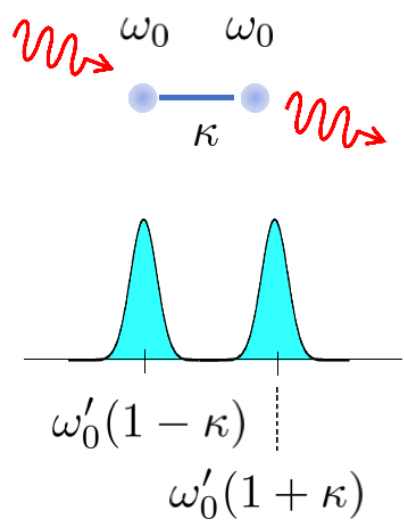

(b)
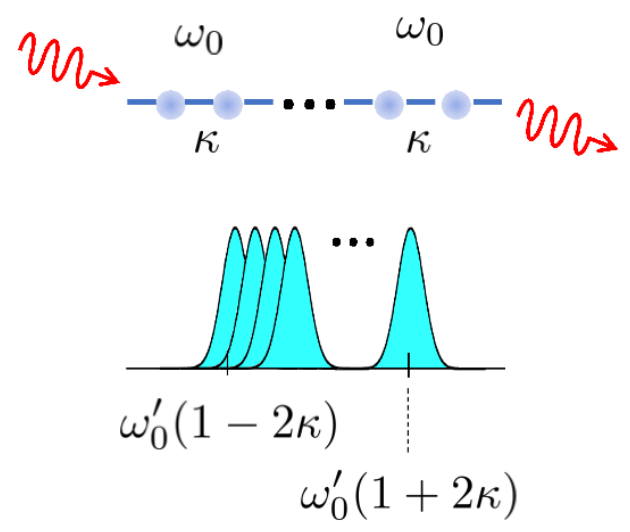

Figure 1 (a) An optical system comprising two identical resonators with inter-resonator coupling coefficient $\mathrm{k}$ exhibits a split transmission spectrum where the separation between the peaks is directly proportional to $\kappa$. (b) A CROW consists of $\mathrm{N}$ coupled resonators and shows a "miniband" transmission characteristic with $\mathrm{N}$ individual super-mode resonances. The bandwidth is again proportional to $\kappa$ but with a different coefficient. 
This NSF-funded project [0642603] is a five-year (60 months, 2007-2012) CAREER (Faculty Early Career Development Program) unified research and education development program. The project's focus is the science, engineering and applications of low-power (milliwatt class) nonlinear optics using CROWs.

In silicon photonics, the main nonlinear optical phenomenon which is relevant at power levels used in optical communications is four-wave mixing (FWM). Because wavelengths shorter than about $1.1 \mu \mathrm{m}$ are absorbed, second-harmonic generation in silicon CROWs [3] of telecommunications band signals is infeasible. Using a strong chi-(3) nonlinearity (which also is the basis of spatial soliton formation [4], [5]), optical pump beams and a signal beam can be made to interact in a fairly short silicon waveguide or compact resonator to generate one or more new frequencies with converted power levels in the tens to hundreds of microwatts. In the traditional context of parametric amplification (of the signal), these new frequencies are called "idlers" but here, they play a central role since they generate a new frequency absent in the plurality of signals at the input to the device. This is more conventionally known as wavelength conversion. The CROW structure is suitable as the foundation of this project because it offers a very high conversion efficiency based on the triple resonance of the pump, signal and idler modes. The enhancement is due to slow-light effects, i.e., a reduction in the group velocity of light. Therefore, studying how the group velocity is affected by dispersion [6] and fabrication disorder in real structures is an essential part of this project.

(a)

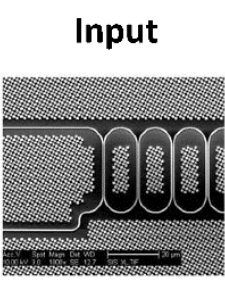

$X 12$ (for 235 rings)

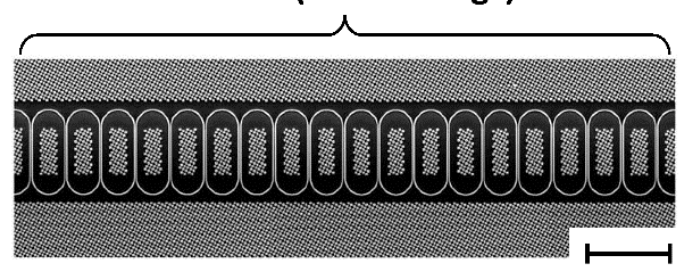

$\longmapsto 30$ um
Output

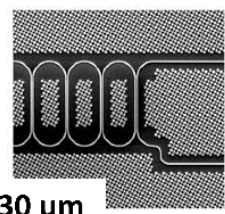

(b)

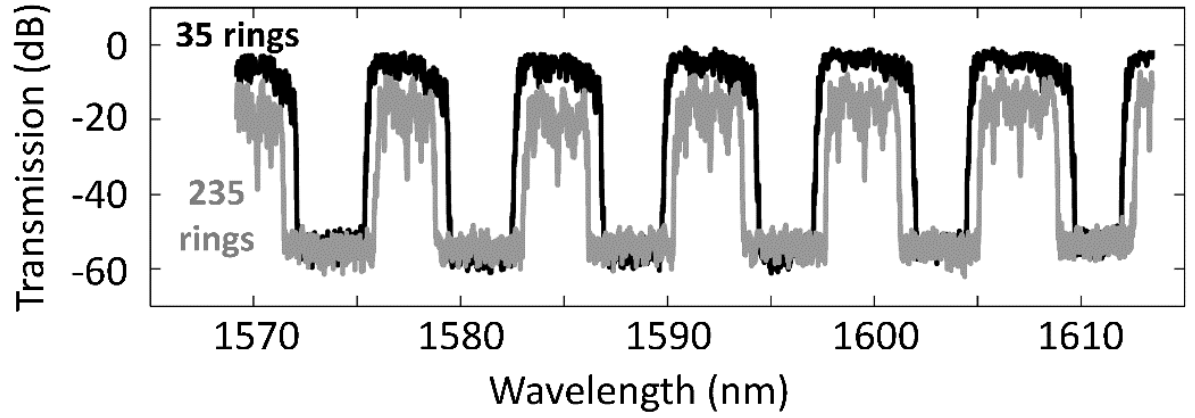

\section{Activities and Accomplishments}


The first set of research activities, labeled Task Area A (Waveguides) in Fig. 1, focuses on improvements and refinements to our knowledge of waveguiding in CROWs. Previously, a tight-binding model of band-center propagation was formulated [7], [8], and theoretical studies of nonlinear propagation were developed on this basis [9], [10]. However, it was predicted that propagation in the band-edge regions of high dispersion and high nonlinearity would be sensitive to disorder [11]. An accurate modeling of waveguiding and dispersion is also necessary for coupled-microring filters [12] or tunable dispersion compensation devices [13].

The first set of activities in this project focused on realizing long CROW structures. We focused on the coupled-microring CROW configuration which was studied by Poon et al. [14] and by IBM [15]. Directional couplers are used to achieve a well-defined coupling coefficient between adjacent unit cells. We experimentally studied the dispersion of directional couplers using a microring coupled to a waveguide [16]. To help in designing structures in silicon photonics, we developed an extension of coupled-mode theory which is applicable to directional couplers in high index contrast materials, in coupledwaveguide structures [17] and coupled-resonator structures [18]. When the optical power is increased, silicon microring resonators with directional couplers can exhibit interesting behavior, such as bistability and nonlinear onoff switching [19]. However, these effects are not very significant at lower power levels.

Figure 2 shows images of sections of coupled-microring CROWs fabricated in this project in collaboration with IBM with several loss-reduction processing steps such as oxidation smoothening of sidewalls and hydrogen annealing. CROWs of up to 235 coupled resonators were successfully fabricated and measured, and a good agreement was achieved between experiments and theory [20]-[22]. Propagation losses as low as $0.062 \mathrm{~dB} /$ ring were measured. Figure 2(b) compares the transmission spectrum of a 35-ring CROW and a 235-ring CROW. The increased passband loss, ripple and band-narrowing are effects of disorder in the CROW, which we studied in detail during this project [22]-[24].

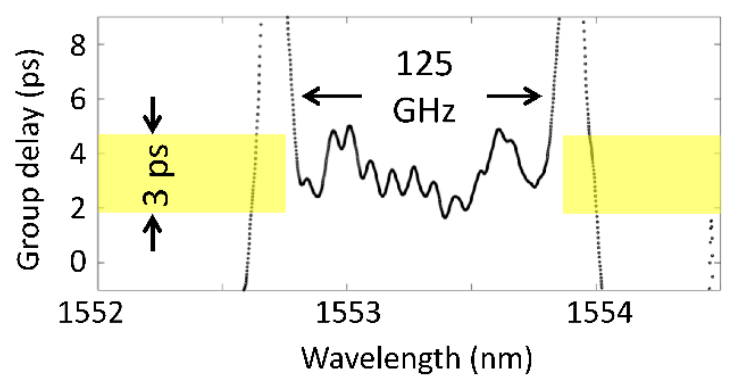

Figure 3 High-resolution measurement of group delay showing the ripple across the transmission band of a 35-ring CROW.
Figure 3 shows an example of a measurement of group delay ripple (GDR) measured for a 35-ring CROW using a highresolution optical vector analyzer. This shows that a reasonably low value of GDR was achieved, less than 3 ps over the passband width of 125 GHz. Consequently, highspeed data modulated signals 
(20 Gb/s NRZ modulation) could be transmitted through CROWs (even as long as 235 coupled resonators) without distortion (see Year 4 report).

(a)

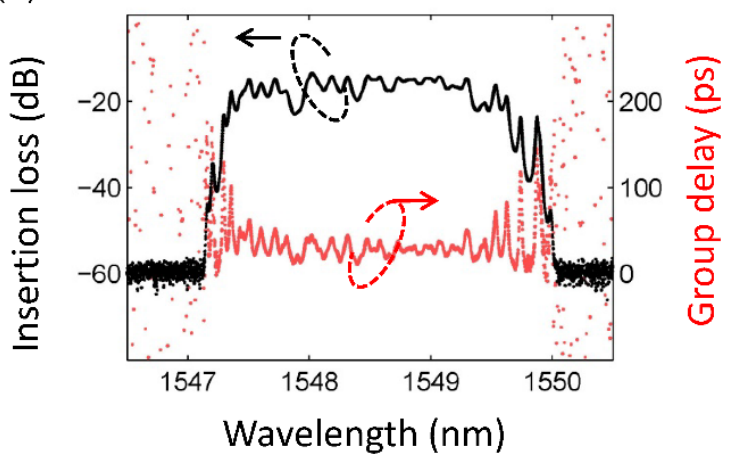

(b)

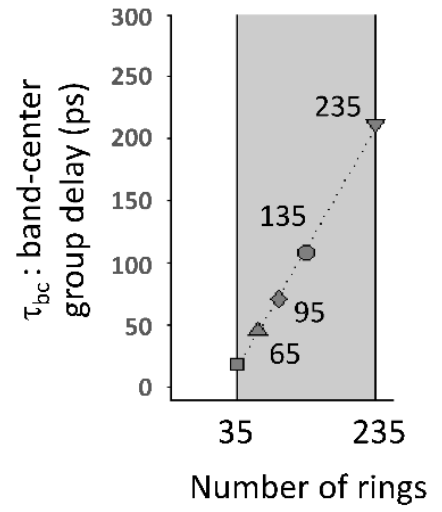

Figure 4 (a) For a 35-ring CROW, TE-polarized transmission (insertion loss) spectrum, i.e., the drop port response normalized to the input port (black), and the group delay spectrum (red). A single CROW band is shown here. (b) Measured band-center group delay $\tau_{\mathrm{bc}}$ for a single CROW band increases linearly with the number of resonators, $\mathrm{N}$

Figure 4 shows a measurement of a transmission band for a 35-ring CROW. Despite some ripple, the band is well defined and has a flat top as is desirable. A total of 600,000 measurements of group delay were analyzed statistically to examine the group delay ripple (GDR) over the central portion of the band. The average band-center group delay $\tau_{\mathrm{bc}}$ was obtained by averaging the root-meansquared group delay, measured with $1.4 \mathrm{pm}$ resolution, over the central onehalf region of each transmission band, which was about $3 \mathrm{~nm}$ wide. As shown in Fig. $4(b), \tau_{b c}$ increased linearly with $N$, the number of resonators.

(a)

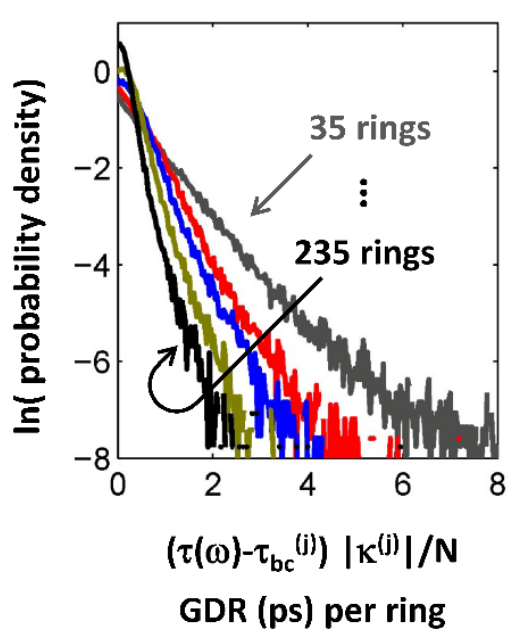

(b)

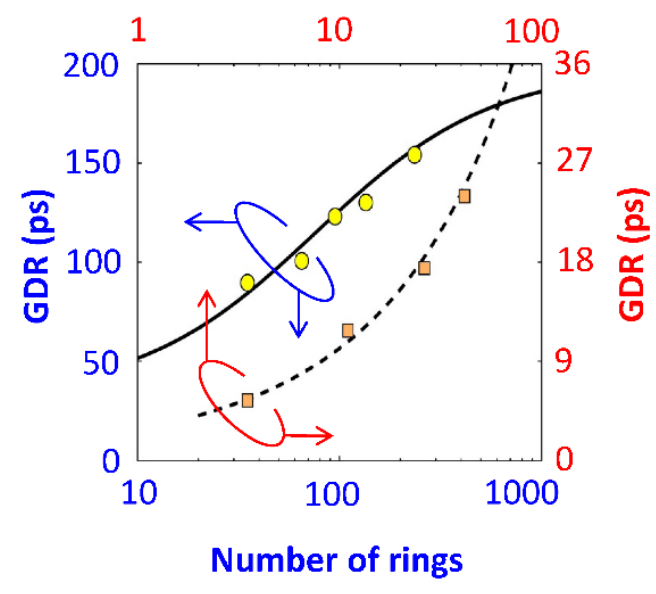

Figure 5(a) Probability density functions (PDFs) of the normalized group delay ripple (GDR) (shown for positive GDR, similar distributions for negative GDR). The PDFs have exponential tails whose slope decreases linearly with the number of resonators. (b) Measured group delay ripple (GDR): 2x standard deviation of the raw GDR data for CROWs is plotted with circles, 
and for waveguides with squares. The dashed line is a $\mathrm{L} 1 / 2$ fit to the waveguide data. The solid line is the predicted GDR for CROWs obtained from PDFs shown in panel (a).

There is valuable information that can be obtained from studying the group delay ripple in more detail. We statistically analyzed measurements of the normalized GDR metric (ps/ring), $\Theta=(\Delta \tau / \mathrm{N})|\kappa|$ where $\Delta \tau$ is the difference between the measured group delay (avoiding the band-edges) and the corresponding band's averaged value, and $|\kappa|$ is the coupling coefficient of that band, derived from the measured bandwidth. As Fig. 2b shows, the distribution of $\Theta$ is well-modeled by an exponential distribution, i.e., $\operatorname{pdf}(|\Theta|) \sim \exp (-$ $\nu \Delta \tau|\kappa| / N)$. Therefore, GDR (amplitude) can be described by twice the standard deviation of the distribution, i.e., by $2 v^{-1}$, which has units of (ps/ring). We have measured that $\lambda$ has a linear dependence on $\mathrm{N}$, with slope $\eta=0.012 \mathrm{ps}^{-1}$. The GDR amplitude in an N-ring CROW was therefore given by $2 \mathrm{Nv}^{-1}(\mathrm{ps})$.

Figure 5(b) shows the group delay ripple amplitude (GDR) for different lengths of CROWs, and also for different lengths of conventional silicon waveguides (without resonators). Whereas the GDR in long CROWs approached an asymptotic upper-bound value, the measured GDR in conventional nanophotonic silicon waveguides (fabricated on the same chip as the CROW devices) increased as $\mathrm{L}^{1 / 2}$, which is similar to the scaling of GDR in cascaded fiber Bragg gratings. This latter behavior arises from incoherent addition of group delay statistics. In contrast, the effective strength of disorder in CROWs is divided by $\mathrm{N}^{1 / 2}$ which compensates the observed $\mathrm{L}^{1 / 2}$ scaling with length in conventional waveguides. Thus, the GDR amplitude in an N-ring CROW, $2 \mathrm{Nv}^{-}$ ${ }^{1}$, shown with a solid black line in Fig. 5(b), reached a constant value, in the large $\mathrm{N}$ limit, equal to $\eta^{-1}$ plus an offset for the other components in the measurement path $=198$ ps (in this batch of devices). Therefore, CROW modes were confirmed experimentally, for the first time, to be phase-coherent collective excitations using high-resolution transmission measurements and a statistic analysis of photon transport.

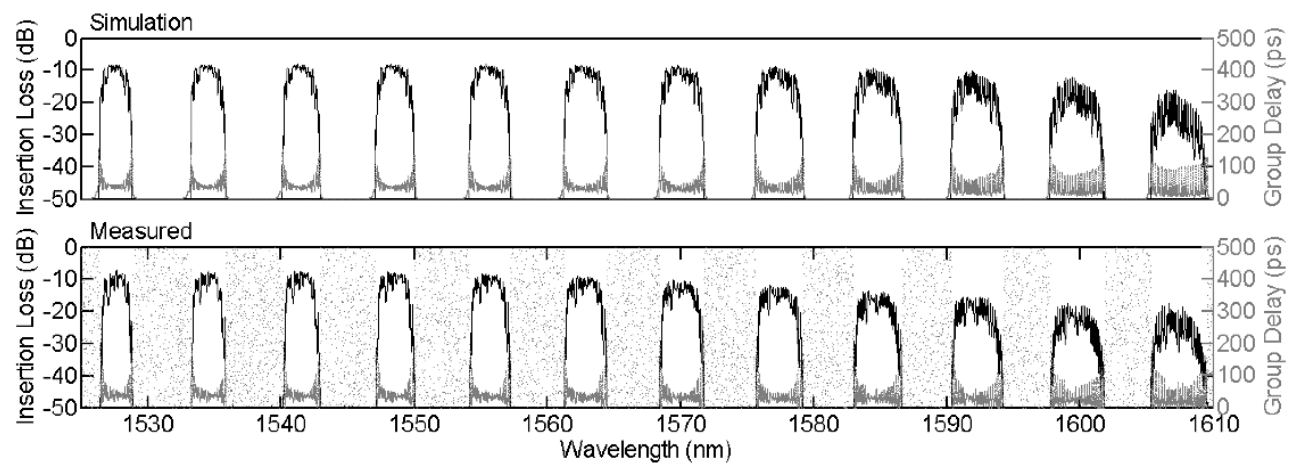

Figure 6 (top panel) simulated transmission spectrum (left axis) and group delay spectrum (right axis) for a silicon microring CROW with disorder. (bottom panel) experimental measurement. 
In addition to the experimental studies, we developed our modeling tools. Traditional simulation methods such as finite-difference time-domain are not well suited to modeling low CROWs with disorder. The length of the structure can be several millimeters, and disorder is manifest at the nanoscale. Therefore, efficient new methods for studying and modeling such structures were developed [22]. An exponential tail of the distribution of time delay and the distribution of GDR was confirmed by numerical simulations of intentionally disordered microring CROWs. Our models were able to accurately reproduce the free-spectral range, transmission band width and amplitude, group delay and even the group delay ripple and amplitude ripple characteristics, as shown in Figure 6.

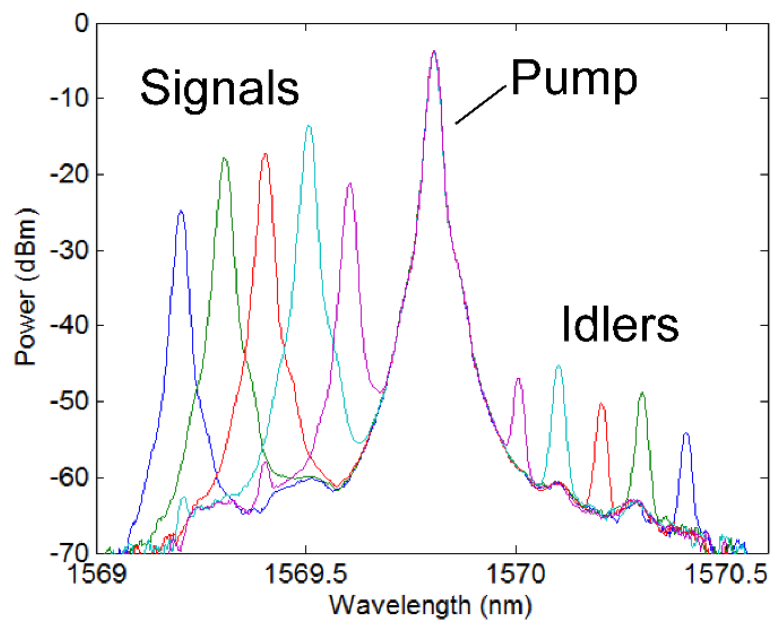

Figure 7 Intra-band four-wave mixing in a 35-ring CROW with signal detuning from -0.2 to $0.6 \mathrm{~nm}$.

The second major set of research activities in this project focused on wavelength conversion using four-wave mixing (FWM). Previously, FWM was studied in silicon waveguides showing its potential for use in optical communications [25]. Here, we studied FWM in silicon microring CROWs. Figure 7 shows the spectra for a group of five measurements, in which a tunable signal was mixed with a fixed-wavelength pump in a 35-ring CROW to generate idlers on the long-wavelength side of the pump. All the wavelengths are within a single passband. The variation in CROW conversion efficiency can be correlated with ripples in the transmission spectrum of the as-fabricated waveguide.

Figure 8 shows the spectra for inter-band FWM, in which the pump and the signals are in different passbands, separated by one free-spectral range. Energy transfer occurs to both higher and lower wavelengths. Once again, the ripples 
in the transmission spectrum of the waveguide lead to variations in the conversion efficiency.

In order to extract the conversion enhancement due to the CROW from the feeder and output waveguides, the conversion efficiency was measured for a straight silicon waveguide (SWG) of the same geometric length. The results are shown in Figure 9. Approximately $+8 \mathrm{~dB}$ and $+5 \mathrm{~dB}$ resonator-enhanced conversion improvements over the straight waveguide were measured for the inter-band and intra-band mixing cases. However, in absolute terms, these conversion efficiencies are not high enough for practical use. In later research, much higher FWM efficiencies were obtained using a single silicon microring resonator [26].

Idlers Signals Pump Idlers

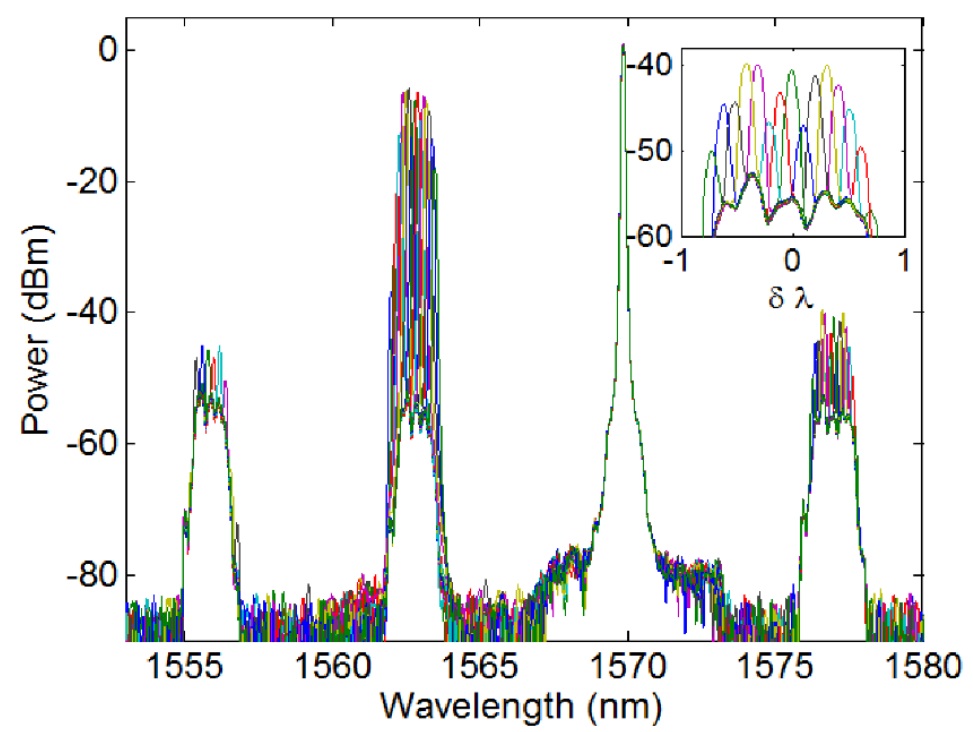

Figure 8 Inter-band four-wave mixing in a 35-ring CROW with signal detuning with respect to pump of -6.3 to $-7.6 \mathrm{~nm}$.

External Collaboration: A collaboration with IBM's silicon photonics group (Dr. W. M. J. Green) provided insights into silicon photonics fabrication and also supported technical collaboration on fabricating the CROW structures [23], [27], [20]. Dr. Ivan B. Divliansky (Research Scientist, CREOL, Florida) collaborated with us for the electron-beam lithography of long waveguide structures [28].

\section{Progress and Impact}

For several years, CROWs remained mainly a theoretical concept, with few experimental demonstrations of more than a dozen or so coupled resonators which showed high loss, lack of tunability and limited practical utility in optical 
communications or signal processing. Around 2007, silicon microring CROWs were studied by IBM for applications in delay lines and optical buffers [15], but were found to be significantly impacted by loss, disorder and dispersion [29]. Since nonlinear optics is even more sensitive to these issues, there had been no intensive study of nonlinear optics in CROWs.

(a)

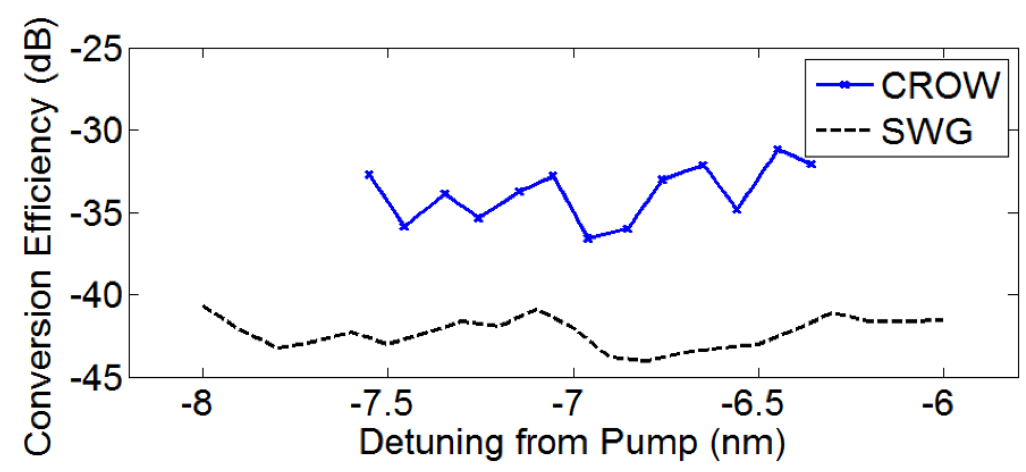

(b)

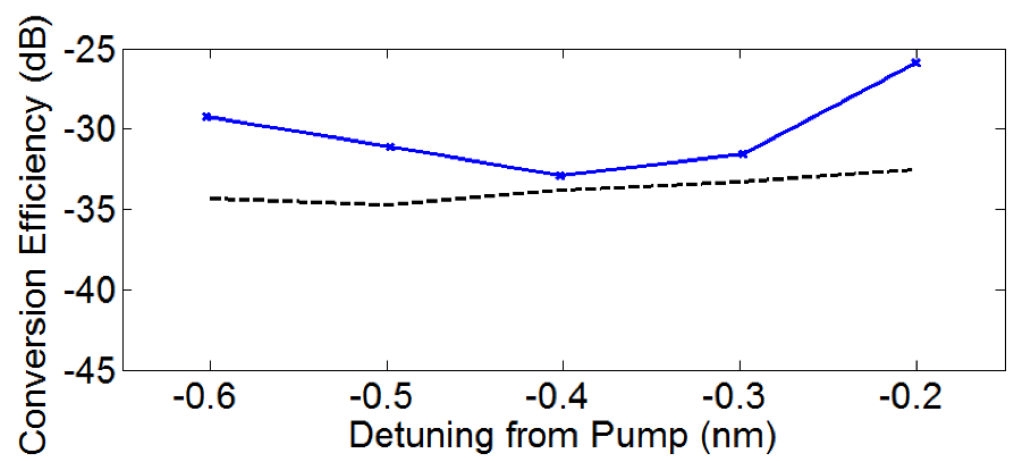

Figure 9 a) Inter-band four-wave mixing: Conversion efficiency of the CROW was about $+8 \mathrm{~dB}$ higher than for a straight waveguide of equivalent geometric length. (b) Intra-band four-wave mixing: Conversion efficiency of the CROW was about $+5 \mathrm{~dB}$ higher than for a straight waveguide of equivalent geometric length.

Prior to this project, there were few experimental reports on long CROWs even in the linear propagation regime, and computational methods had not yet been developed and validated which could calculate optical propagation realistically in CROWs that comprise several hundred coupled resonators. We made advances in all these areas by studying and modeling disorder effects, improving fabrication technology and demonstrating four-wave mixing in CROWs.

Once disorder, loss and dispersion are partially mitigated, the benefits of the CROW device architecture for integrated nonlinear optics become apparent. For example, for a typical silicon single-mode waveguide at $1550 \mathrm{~nm}$, the fourwave mixing nonlinear coefficient is $\gamma=200 \mathrm{~W}^{-1} \mathrm{~m}^{-1}$. A 35-ring CROW was experimentally measured to have a slow-light enhanced value $\gamma_{\text {eff }}=4150 \mathrm{~W}^{-1}$ 
$\mathrm{m}^{-1}$. Since the wavelength-converted power (idler) in FWM is quadratically proportional to $\gamma$, the improvement in $\gamma$ by a factor of about 50 compared to a conventional waveguide of the same length leads to a $34 \mathrm{~dB}$ improvement in the converted power at the new wavelength. However, it is easier to make conventional waveguides longer, since the length of CROWs has been difficult to scale beyond a few hundred resonators.

In addition to classical FWM, we developed a model for spontaneous four-wave mixing (SFWM) using the Collett-Gardiner approach [30]. This method formulates the time-domain equations of motion in the Heisenberg picture. We solved the equations in the case of a single resonator as well as for multiple resonators, showing the potential advantages of the latter.

Impact on Human Resources: Three graduate students in the PI's group worked on this topic as part of their education and training. A post-doctoral scholar, although not directly supported by CAREER funds, has contributed to the CROW measurements and is a co-author on the relevant papers. Students presented papers at a number of major conferences including CLEO.

A graduate student interacted on a weekly basis with under-privileged children of the Preuss School from disadvantaged families; these students must qualify for Federal Lunch Programs and be from families that have no college education. The mentorship was valuable in encouraging these students to enroll for college in science and engineering majors.

\section{Subsequent Extensions}

Research in this project accomplished a significant improvement in the stateof-the-art of CROW waveguides. Low-loss CROW waveguides of record length were demonstrated in using coupled microrings in silicon photonics, and four-wave mixing was demonstrated. Based on the outcomes of this CAREER project, a wide range of subsequent projects were initiated on focused research topics, including improved nonlinear mixers, harnessing disorder for functionality, and quantum photonics. In some of these projects, we continued to use CROWs. In some cases, single microring resonators with a higher quality factor offered better performance in experiments.

Infrared imaging is another useful experimental tool which we developed during this project, to study light propagation through the length of these structures [28], [31]. Light from a tunable laser is transmitted through the device and the device plane is imaged using a microscope with a high numerical aperture objective and an InGaAs camera. Images are taken at different wavelengths, and in principle, contain the same information that is obtained by transmission measurements made using optical fibers. However, typical infrared images of guided light at $1550 \mathrm{~nm}$ lack the quality and depth of 
information needed to perform quantitative analysis, such as extracting propagation loss, or coupling coefficients. We developed an experimental procedure which allows us to achieve high dynamic range infrared imaging of light propagation by "bracketing" multiple image acquisitions made at different exposure settings.

The CROW structures studied in this project are passive waveguide structures. A tunable laser was used to study optical propagation at different points in the dispersion relationship. In the future, we seek a control "knob" to tune the waveguiding properties of the CROW structure without changing the wavelength of the laser. The most practical way of tuning silicon microring CROWs is to use biasing of a p-n junction [32]. Integrated electro-optic modulators could be another way of achieving phase shifters with more than $100 \mathrm{GHz}$ of bandwidth, as was demonstrated later [33]. The thermo-optic effect, which we used for tunable filters, is also another option as a control knob that can be used to control silicon photonics devices over a wide bandwidth [34]. However, it is difficult to tune individual rings in a CROW structure using this approach since heat spreading can lead to crosstalk.

The CROW structure can have other applications including generation of quantum light [30], and developing amplifiers and lasers with novel properties [35]. The resonator-enhanced nonlinearity benefits the generation of entangled photon-pair and heralded single-photon generation using silicon photonics [36]. Unlike slow-light photonic crystal waveguides, a CROW provides enhanced nonlinearity in several passbands simultaneously which can be useful for multiplexed photon-pair generation [37] and tuning the entanglement properties [38]. Our initial measurements of spontaneous four-wave mixing (SFWM) were on correlated photon-pair generation and heralded single-photon generation. An experimental measurement of entanglement was performed later, using a two-photon interferometer constructed to verify time-energy entanglement [36]. Going beyond what was achieved using CROWs, bright and high quality photon-pair generation and heralded single-photon generation was shown later using high-Q single silicon microring resonators [39].

Microring resonators are sensitive to disorder. We studied potential methods for precisely tuning resonators without heaters. Our method is based on fieldinduced local oxidation of $\mathrm{Si}$ to $\mathrm{SiO}_{2}$ via a chemical reaction near an electrically-biased conducting atomic-force microscope tip [40], [41]. Scanning probe lithography has previously been used to modify the resonance frequency of a GaAs photonic crystal cavity. A single silicon microresonator can be monitored and controlled more easily [42] than a long CROW device.

\section{Open-Access Reporting Initiative}


PRAISE: This open-access document is provided in support of our PRAISE (Public Report of Activities, Impact and Subsequent Extensions) initiative. What is it? An open-access document shared with the public which describes the research outcomes of publicly-funded projects. For us, these projects are typically funded by the NSF (National Science Foundation).

\section{References}

[1] A. Yariv, Y. Xu, R. K. Lee, and A. Scherer, "Coupled-resonator optical waveguide: a proposal and analysis," Opt. Lett., vol. 24, no. 11, pp. 711$713,1999$.

[2] N. Stefanou and A. Modinos, "Impurity bands in photonic insulators," Phys. Rev. B, vol. 57, no. 19, pp. 12127-12133, May 1998, doi: 10.1103/PhysRevB.57.12127.

[3] S. Mookherjea and A. Yariv, "Second-harmonic generation with pulses in a coupled-resonator optical waveguide," Phys. Rev. E, vol. 65, no. 2, p. 026607, Jan. 2002, doi: 10.1103/PhysRevE.65.026607.

[4] A. Ciattoni, B. Crosignani, S. Mookherjea, and A. Yariv, "Nonparaxial dark solitons in optical Kerr media," Opt. Lett., vol. 30, no. 5, p. 516, Mar. 2005, doi: 10.1364/OL.30.000516.

[5] B. Crosignani, A. Yariv, and S. Mookherjea, "Nonparaxial spatial solitons and propagation-invariant pattern solutions in optical Kerr media," Opt. Lett., vol. 29, no. 11, p. 1254, Jun. 2004, doi: 10.1364/OL.29.001254.

[6] S. Mookherjea, "Dispersion characteristics of coupled-resonator optical waveguides," Opt. Lett., vol. 30, no. 18, p. 2406, Sep. 2005, doi: 10.1364/OL.30.002406.

[7] S. Mookherjea and A. Yariv, "Optical pulse propagation in the tightbinding approximation," Opt. Express, vol. 9, no. 2, p. 91, Jul. 2001, doi: 10.1364/OE.9.000091.

[8] S. Mookherjea and A. Yariv, "Pulse propagation in a coupled resonator optical waveguide to all orders of dispersion," Phys. Rev. E, vol. 65, no. 5, p. 056601, Apr. 2002, doi: 10.1103/PhysRevE.65.056601.

[9] S. Mookherjea and A. Yariv, "Kerr-stabilized super-resonant modes in coupled-resonator optical waveguides," Phys. Rev. E, vol. 66, no. 4, p. 046610, Oct. 2002, doi: 10.1103/PhysRevE.66.046610.

[10] S. Mookherjea and A. Yariv, "Optical pulse propagation and holographic storage in a coupled-resonator optical waveguide," Phys. Rev. $E$, vol. 64 , no. 6, p. 066602 , Nov. 2001, doi: 10.1103/PhysRevE.64.066602.

[11] S. Mookherjea, D. S. Cohen, and A. Yariv, "Nonlinear dispersion in a coupled-resonator optical waveguide," Opt. Lett., vol. 27, no. 11, p. 933, Jun. 2002, doi: 10.1364/OL.27.000933. 
[12] J. R. Ong, R. Kumar, and S. Mookherjea, "Silicon microring-based wavelength converter with integrated pump and signal suppression," Opt. Lett., vol. 39, no. 15, p. 4439, Aug. 2014, doi: 10.1364/OL.39.004439.

[13] S. Mookherjea, "Using gain to tune the dispersion relation of coupledresonator optical waveguides," IEEE Photon. Technol. Lett., vol. 18, no. 5, pp. 715-717, Mar. 2006, doi: 10.1109/LPT.2006.871144.

[14] J. Poon, J. Scheuer, S. Mookherjea, G. T. Paloczi, Y. Huang, and A. Yariv, "Matrix analysis of microring coupled-resonator optical waveguides," Opt. Express, vol. 12, no. 1, p. 90, 2004, doi: 10.1364/OPEX.12.000090.

[15] F. Xia, L. Sekaric, and Y. Vlasov, "Ultracompact optical buffers on a silicon chip," Nature Photon, vol. 1, no. 1, pp. 65-71, Jan. 2007, doi: 10.1038/nphoton.2006.42.

[16] R. Aguinaldo, Yiran Shen, and S. Mookherjea, "Large Dispersion of Silicon Directional Couplers Obtained via Wideband Microring Parametric Characterization," IEEE Photon. Technol. Lett., vol. 24, no. 14, pp. 1242-1244, Jul. 2012, doi: 10.1109/LPT.2012.2198639.

[17] M. L. Cooper and S. Mookherjea, "Numerically-assisted coupledmode theory for silicon waveguide couplers and arrayed waveguides," Opt. Express, vol. 17, no. 3, p. 1583, Feb. 2009, doi: 10.1364/OE.17.001583.

[18] S. Mookherjea, "Spectral characteristics of coupled resonators," $J$. Opt. Soc. Am. B, vol. 23, no. 6, p. 1137, Jun. 2006, doi: 10.1364/JOSAB.23.001137.

[19] S. Mookherjea and M. A. Schneider, "The nonlinear microring adddrop filter," Opt. Express, vol. 16, no. 19, p. 15130, Sep. 2008, doi: 10.1364/OE.16.015130.

[20] M. L. Cooper et al., "235-ring Coupled-Resonator Optical Waveguides," in Conference on Lasers and Electro-Optics 2010, San Jose, California, 2010, p. CTuHH3. doi: 10.1364/CLEO.2010.CTuHH3.

[21] S. Mookherjea and M. A. Schneider, "Avoiding bandwidth collapse in long chains of coupled optical microresonators," Opt. Lett., vol. 36, no. 23, p. 4557, Dec. 2011, doi: 10.1364/OL.36.004557.

[22] M. L. Cooper and S. Mookherjea, "Modeling of Multiband Transmission in Long Silicon Coupled-Resonator Optical Waveguides," IEEE Photon. Technol. Lett., vol. 23, no. 13, pp. 872-874, Jul. 2011, doi: 10.1109/LPT.2011.2141657.

[23] M. L. Cooper et al., "Waveguide dispersion effects in silicon-oninsulator coupled-resonator optical waveguides," Opt. Lett., vol. 35, no. 18, p. 3030, Sep. 2010, doi: 10.1364/OL.35.003030.

[24] M. L. Cooper et al., "Statistics of light transport in 235-ring silicon coupled-resonator optical waveguides," Opt. Express, vol. 18, no. 25, p. 26505, Dec. 2010, doi: 10.1364/OE.18.026505. 
[25] R. Salem, M. A. Foster, A. C. Turner, D. F. Geraghty, M. Lipson, and A. L. Gaeta, "Signal regeneration using low-power four-wave mixing on silicon chip," Nature Photon, vol. 2, no. 1, pp. 35-38, Jan. 2008, doi: 10.1038/nphoton.2007.249.

[26] J. R. Ong, R. Kumar, R. Aguinaldo, and S. Mookherjea, "Efficient CW Four-Wave Mixing in Silicon-on-Insulator Micro-Rings With Active Carrier Removal," IEEE Photon. Technol. Lett., vol. 25, no. 17, pp. 16991702, Sep. 2013, doi: 10.1109/LPT.2013.2272521.

[27] J. R. Ong et al., "Low-power continuous-wave four-wave mixing in silicon coupled-resonator optical waveguides," Opt. Lett., vol. 36, no. 15, pp. 2964-2966, 2011.

[28] M. L. Cooper, G. Gupta, J. S. Park, M. A. Schneider, I. B. Divliansky, and S. Mookherjea, "Quantitative infrared imaging of silicon-on-insulator microring resonators," Opt. Lett., vol. 35, no. 5, p. 784, Mar. 2010, doi: 10.1364/OL.35.000784.

[29] J. B. Khurgin, "Dispersion and loss limitations on the performance of optical delay lines based on coupled resonant structures," Opt. Lett., vol. 32, no. 2, p. 133, Jan. 2007, doi: 10.1364/OL.32.000133.

[30] J. R. Ong and S. Mookherjea, "Quantum light generation on a silicon chip using waveguides and resonators," Opt. Express, vol. 21, no. 4, p. 5171, Feb. 2013, doi: 10.1364/OE.21.005171.

[31] S. Mookherjea and H. R. Grant, "High dynamic range microscope infrared imaging of silicon nanophotonic devices," Opt. Lett., vol. 37, no. 22, p. 4705, Nov. 2012, doi: 10.1364/OL.37.004705.

[32] S. Mookherjea, J. R. Ong, X. Luo, and L. Guo-Qiang, "Electronic control of optical Anderson localization modes," Nature Nanotech, vol. 9, no. 5, pp. 365-371, May 2014, doi: 10.1038/nnano.2014.53.

[33] X. Wang, P. O. Weigel, J. Zhao, M. Ruesing, and S. Mookherjea, "Achieving beyond-100-GHz large-signal modulation bandwidth in hybrid silicon photonics Mach Zehnder modulators using thin film lithium niobate," APL Photonics, vol. 4, no. 9, p. 096101, Sep. 2019, doi: 10.1063/1.5115243.

[34] R. Aguinaldo et al., "Wideband silicon-photonic thermo-optic switch in a wavelength-division multiplexed ring network," Opt. Express, vol. 22, no. 7, p. 8205, Apr. 2014, doi: 10.1364/OE.22.008205.

[35] S. Mookherjea, "Semiconductor coupled-resonator optical waveguide laser," Appl. Phys. Lett., vol. 84, no. 17, pp. 3265-3267, Apr. 2004, doi: 10.1063/1.1719278.

[36] R. Kumar, M. Savanier, J. R. Ong, and S. Mookherjea, "Entanglement measurement of a coupled silicon microring photon pair source," Opt. Express, vol. 23, no. 15, p. 19318, Jul. 2015, doi: 10.1364/OE.23.019318.

[37] R. Kumar, J. R. Ong, J. Recchio, K. Srinivasan, and S. Mookherjea, "Spectrally multiplexed and tunable-wavelength photon pairs at $155 \mu \mathrm{m}$ 
from a silicon coupled-resonator optical waveguide," Opt. Lett., vol. 38, no. 16, p. 2969, Aug. 2013, doi: 10.1364/OL.38.002969.

[38] R. Kumar, J. R. Ong, M. Savanier, and S. Mookherjea, "Controlling the spectrum of photons generated on a silicon nanophotonic chip," Nat Commun, vol. 5, no. 1, p. 5489, Dec. 2014, doi: 10.1038/ncomms6489.

[39] C. Ma, X. Wang, V. Anant, A. D. Beyer, M. D. Shaw, and S. Mookherjea, "Silicon photonic entangled photon-pair and heralded single photon generation with CAR $>12,000$ and $\mathrm{g}^{\wedge}(2)(0)<0006$," Opt.

Express, vol. 25, no. 26, p. 32995, Dec. 2017, doi: 10.1364/OE.25.032995.

[40] Y. Shen, I. B. Divliansky, D. N. Basov, and S. Mookherjea, "Perfect set-and-forget alignment of silicon photonic resonators and interferometers," in Optical Fiber Communication Conference/National Fiber Optic Engineers Conference 2011, Los Angeles, California, 2011, p. PDPC3. doi: 10.1364/OFC.2011.PDPC3.

[41] Y. Shen, I. B. Divliansky, D. N. Basov, and S. Mookherjea, "Electricfield-driven nano-oxidation trimming of silicon microrings and interferometers," Opt. Lett., vol. 36, no. 14, p. 2668, Jul. 2011, doi: 10.1364/OL.36.002668.

[42] M. Savanier, R. Kumar, and S. Mookherjea, "Optimizing photon-pair generation electronically using a $p-i-n$ diode incorporated in a silicon microring resonator," Appl. Phys. Lett., vol. 107, no. 13, p. 131101, Sep. 2015, doi: 10.1063/1.4932047. 